\title{
Company Index
}

\section{A Guide to Companies}

Abberior Instruments America

Absolute Clarity \& Calibration, LLC

Access Optics

ACCU-SCOPE Inc.

Adsys Controls, Inc.

Advanced MicroBeam, Inc.

Advanced Microscopy Techniques, Inc.

Advance Surface Microscopy

Advent Research Materials

Aerotech, Inc.

Agilent Technologies

Alliance Technologies

Alluxa Inc.

Alrad

Amptek, Inc.

Anatech USA

Anderson Materials Evaluation

Andor Technology

Angstrom Scientific Inc.

Applied Beams LLC

Applied Physics Technologies, Inc.

Applied Thermal Control Ltd

Archer OpTx, Inc.

Argolight

ASI/Applied Scientific Instrumentation

Asylum Research

Attocube Systems AG

B \& W Tek, Inc.

Basler Inc.

BioTek Instruments, Inc.

Black Mesa Imaging, LLC

Bock Optronics

Boston Electronics

BrandTech Scientific, Inc.

Bruker Nano Analytics www.abberior-instruments-america.com

www.absoluteclarity.com

www.accessoptics.com

www.accu-scope.com

www.AdsysControls.com

www.advancedmicrobeam.com

www.amtimaging.com

www.asmicro.com

www.advent-rm.com

www.aerotech.com

www.agilent.com/chem/ilab

www.alliancetechgroup.com

www.alluxa.com

www.alrad.com

www.amptek.com

www.anatechusa.com

www.andersonmaterials.com

www.andor.com

www.angstrom.us

www.appliedbeams.com

p. 3

www.a-p-tech.com

p. 4

www.app-therm.com

www.archeroptx.com

www.argolight.com

www.asiimaging.com

p. 5

www.oxford-instruments.com/AFM

www.attocube.com

www.bwtek.com

www.baslerweb.com

p. 6

www.biotek.com

http://blackmesa-imaging.com

www.bockoptronics.ca

www.boselec.com

www.brandtech.com

www.bruker.com/nano-analysis 


\section{A Guide to Companies}

Bruker Nano Surfaces

Bulbworks, Inc.

Bunton Instrument Co.

Cadmet Inc.

CAMECA

Capovani Brothers Inc.

Carbon Design Innovations

Charles Sheets

CoolLED Limited

Coxem Co., Ltd

CPGlobalTech

CRAIC Technologies

Cryofab, Inc.

CytoViva, Inc.

Czitek

Deben

DELMIC BV

Delong America Inc.

Denton Vacuum, LLC

Diatome U.S.

Digital Surf

Dino-Lite Scopse (BigC)

Direct Electron, LP

Duniway Stockroom Corp.

\section{Ealing Catalog}

EDAX, Inc.

Edge-3d

Electron Microscopy Sciences

Electron Optica, Inc.

Electro-Optics Technology, Inc.

EMSIS GmbH

Energetiq Technology

Euresys Inc

Evactron by XEI Scientific

Excelitas Technologies www.bruker.com

p. 22

www.bulbworks.com

www.buntgrp.com

www.cadmet.com

www.cameca.com

www.capovani.com

www.carbondesigninnovations.com

www.gamma-sci.com

www.coolled.com

www.coxem.com

p. 7

www.globaltech.com

www.microspectra.com

www.cryofab.com

www.cytoviva.com

www.czitek.com

www.deben.co.uk

p. 22

www.delmic.com

www.lv-em.com

www.dentonvacuum.com

www.emsdiasum.com

pp. 8, 22

www.digitalsurf.com

www.dinolite.us

www.directelectron.com

p. 9

www.duniway.com

www.ealingcatalog.com

www.edax.com

p. 23

www.edge-3d.com

www.emsdiasum.com

pp. 10,23

www.electronoptica.com

www.eotech.com

www.emsis.eu

p. 23

www.energetiq.com

p. 23

www.euresys.com

www.evactron.com

p. 23

www.excelitas.com

p. 23 


\section{Company Index}

\section{A Guide to Companies}

EXpressLO LLC

\section{Exprodo Software}

Field Management Services

Filmetrics, Inc.

Finger Lakes Instrumentation

Fischer Technology, Inc.

FOM Networks, Inc.

Gatan, Inc.

Geller MicroAnalytical Laboratory

General Data Healthcare

GenTech Scientific, Inc.

Glen Mills Inc.

GOYO North America

Haydon Kerk Motion Solutions, Inc.

HEIDENHAIN Corpoation

HelioWorks, Inc.

HEMCO Corporation

Hiden Analytical Limited

Hitachi High Technologies America, Inc.

Hooke College of Applied Sciences

HORIBA Scientific

HREM Research, Inc.

HTA Photomask

Hummingbird Scientific

ibidi, LLC

ibss Group, Inc.

Imaging Solutions Group

International Centre for Diffraction Data (ICDD)

ION-TOF

IXRF Systems, Inc.

J. Kraft Microscopy Services

Janis Research Co.

JC Nabity Lithography Systems

JEOL USA, Inc.

Kammrath and Weiss
www.EXpressLO.com

p. 24

www.expodo.com

www.fms-corp.com

www.filmetrics.com

www.flicamera.com

www.fischer-technology.com

www.fomnetworks.com

www.gatan.com

p. 11

www.gellermicro.com

www.general-data.com

www.gentechscientific.com

www.glenmills.com

www.goyonorthamerica.com

www.haydonkerkpittman.com

www.heidenhain.us

www.helioworks.com

www.hemcocorp.com

www.hidenanalytical.com

www.hitachi-hightech.com/us

www.hookecollege.com

www.horiba.com/scientific

www.hremresearch.com

www.htaphotomask.com

www.hummingbirdscientific.com

www.ibidi.com

www.ibssgroup.com

p. 24

www.isgcameras.com

www.icdd.com

p. 24

www.iontofusa.com

www.ixrfsystems.com

p. 24

WWW.JKRAFTMICRO.COM

p. 12

www.janis.com/microscopy.aspx

www.jcnabity.com

www.jeolusa.com

www.kammrath-weiss.com/en 


\section{Company Index}

\section{A Guide to Companies}

Kapteyn Mumane Laboratories

Kewaunee Scientific Corporation

KEYENCE Corporation

Kimmon Koha USA, Inc.Kimball Physics, Inc.

Kleindiek Nanotechnik

K-Tek Nanotechnology, LLC

Kurt J. Lesker Company

Lambert Instruments

Laser 2000 (UK) Ltd

LatticeGear, LLC

Lehigh Microscopy School

Leica Microsystems, Inc.

Linkam Scientific Instruments

Lumencor, Inc.

Lytron Inc.

M.E. Taylor Engineering, Inc.

Mad City Labs, Inc.

Mager Scientific

McCrone Associates

McCrone Atlas of Microscopic Particles

McCrone Microscopes \& Accessories

McCrone Research Institute

McCrone Research Institute Chicago Division

McPHERSON

Metrigraphics, LLC

Micro Photonics

Microscopy Innovations, LLC

Microtechnics

Microtome Service Company

Midwest Optical Systems

Minitool Inc.

Minus K Technology, Inc.

MLD Technologies, LLC

Motic Instruments Inc

Motion X Corporation www.kmlabs.com

www.kewaunee.com

www.keyence.com

www.kimmon.com

www.kleindiek.com

www.kteknano.com

p. 14

www.lesker.com

www.lambertinstruments.com

www.laser2000.co.uk

www.latticegear.com

www.lehigh.edu/microscopy

www.leica-microsystems.com

www.linkam.co.uk

p. 25

www.lumencor.com

www.Lytron.com

www.semicro.org

www.madcitylabs.com

www.magersci.com

www.mccrone.com

www.mccroneatlas.com

www.mccrone.com

www.mcri.org

www.mcri.org

www.McPhersonlnc.com

www.metrigraphicsllc.com

www.microphotonics.com

www.microscopyinnovations.com

www.microtechnics.com

www.microtomeserviceco.com

p. 25

www.midopt.com

www.minitoolinc.com

p. 25

www.minusk.com

www.mldtech.com

www.motic.com

www.motionxcorp.com 


\section{Company Index}

\section{A Guide to Companies}

Nanolmages, LLC

Navitar, Inc.

Norsam Technologies, Inc.

Olympus Corporation of the Americas

Scientific Solutions Group

Olympus Corporation

Optronics

Oregon Physics

OSI Optoelectronics Inc.

Oxford Instruments America, Inc.

Pantec Engineering AGPark Systems, Inc.

Park Systems

PCO AG

PCO-TECH Inc.

Pfeiffer Vacuum

PHASEVIEW

Phenom-World

Photometrics

Photron

Physical Electronics, Inc.

PI (Physik Instrumente), LP

\section{PIEZOCONCEPT}

PIKE Technologies, Inc.

PNDetector GmbH

Precision Glass \& Optics (PG\&O®)

Protochips, Inc.

Pyramid Imaging, Inc.

QImaging

Quantum Composers Inc.

Quantom Design, Inc.

Rainbow Research Optics, Inc.

Raith America, Inc.

Raptor Photonics

RaySpec Ltd

retroDIODE LLC www.nanoimages.com

www.navitar.com

p. 25

www.norsam.com

www.olympus-lifescience.com

www.olympus-ims.com

www.optronics.com

www.oregon-physics.com

p. 26

www.osioptoelectronics.com

nano.oxinst.com

www.pantec-medicallaser.com

www.parkafm.com

p. 26

www.pco-tech.com

p. 15

www.pco-tech.com

www.pfeiffer-vacuum.com

www.phaseview.com

www.phenom-world.com

p. 16

www.photometrics.com

www.photron.com

www.phi.com

www.pi-usa.us/microscopy

p. 26

www.piezoconcept.com

www.piketech.com

www.pndetector.de

www.pgo.com

www.protochips.com

www.pyramidimaging.com

www.qimaging.com

www.quantumcomposers.com

www.qdusa.com/products/getec_afsem.html

www.RR-Optics.com

www.raith.com

www.raptorphotonics.com

www.rayspec.co.uk

www.retrodiode.com 


\section{Company Index}

\section{A Guide to Companies}

REYNARD CORP

Rigaku Corporation

RMC Boeckeler

SCHOTT North America, Inc. Lighting and Imaging

Scientific Volume Imaging

SemionCompany

SmarAct Inc.

Special Optics

Spectrolight Inc.

SPI Supplies

SPOT Imaging Solutions

STAR Cryoelectronics/H.K.N. Inc.

Sutter Instrument

TCG, Inc.

Ted Pella, Inc.

Tescan USA

Thermo Fisher Scientific

TOFRA, Inc

Toshiba Imaging Systems Division

Tousimis Research Corporation

TREK, INC.

TSS Microscopy

UNITRON Ltd.

US fiberoptec Technology, Inc.

Velmex, Inc.

Vision Engineering Inc.

WITec Instruments Corp. GmbH

XRE

XEI Scientific, Inc.

Z\&Z Optoelectronics Tech Co

ZEISS www.reynardcorp.com

www.rigaku.com

p. 26

www.rmcboeckeler.com

www.us.schott.com/lightingimaging

p. 26

www.svi.nl

www.semionco.com

www.smaract.com

p. 27

www.specialoptics.com

p. 27

www.spectrolightinc.com

www.2spi.com

www.spotimaging.com

www.starcryo.com

www.sutter.com

www.tcg-rep.com

www.tedpella.com

pp. 18,28

www.tescan.com

pp. 19,28

thermofisher.com/msr

www.tofrainc.net

www.toshibacameras.com

www.tousimis.com

www.trekinc.com

www.tssmicroscopy.com

www.unitronusa.com

www.usfiberoptec.com

www.velmex.com

www.visioneng.us

www.witec.de

www.xre.be

(see Evactron by XEI Scientific)

www.zzoptic.com

www.zeiss.com/microscopy

p. 20 

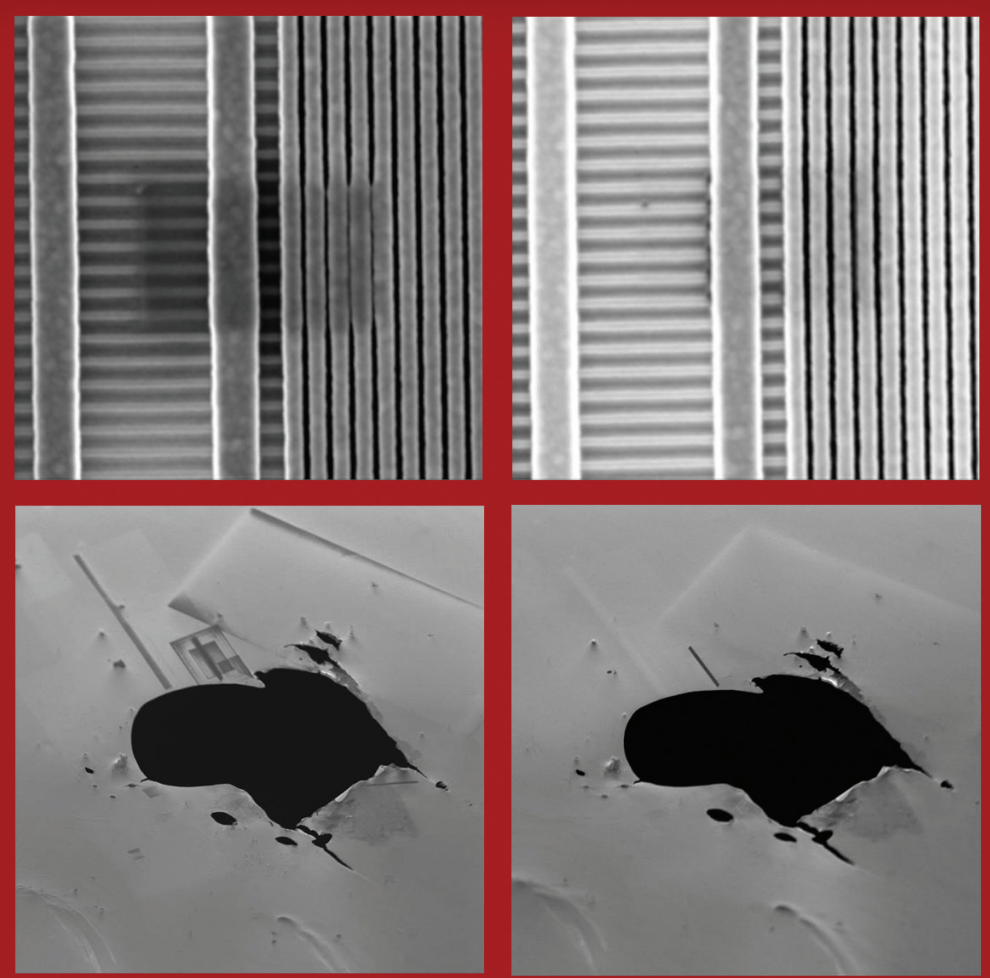

BEFORE

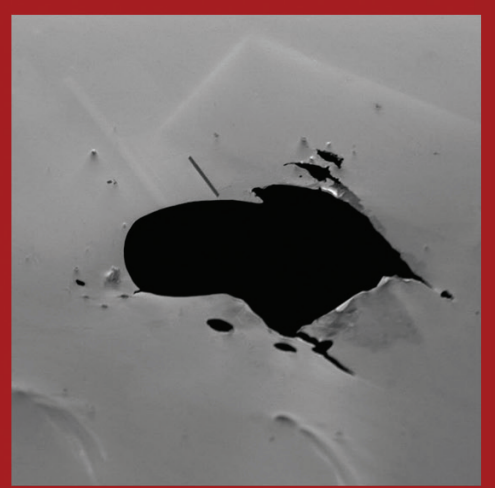

AFTER

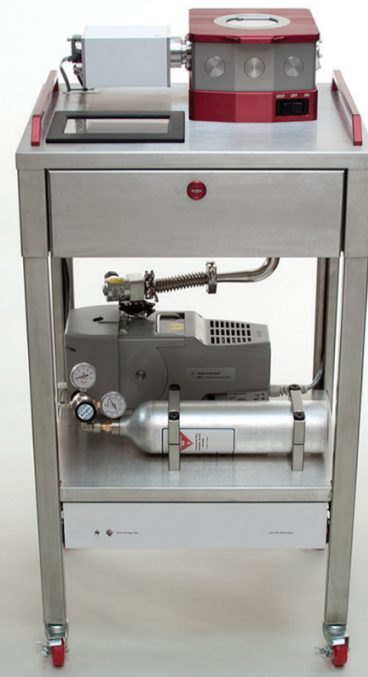

MOBILE CUBIC ASHER ${ }^{\mathrm{TM}}$

A clean image worth a thousand words.

Let your images say more. ibss 